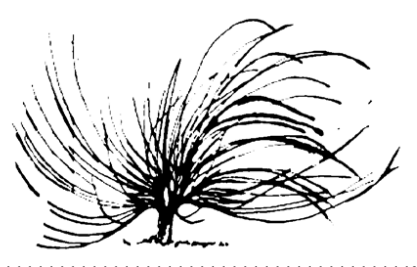

\title{
Elementos resilientes utilizados por estudiantes de la Normal Rural Vasco de Quiroga (Michoacán México) en su movimiento ${ }^{1}$
}

\author{
Cinthia Gricell Arreola Piñón ${ }^{2}$ \\ Universidad de Morelia \\ México \\ citlali.29@hotmail.com \\ María Inés Gómez del Campo del Paso ${ }^{3}$ \\ Universidad Michoacana de San Nicolás de Hidalgo \\ México \\ igomezdelcampo@hotmail.com
}

\begin{abstract}
Resumen
La presente investigación tiene por objetivo describir los recursos resilientes que emplean los estudiantes de la Escuela Normal Rural Vasco de Quiroga en su movimiento estudiantil y en su formación profesional. Esta investigación se realizó a través de una metodología cualitativa de tipo fenomenológico, con la participación de 40 estudiantes varones, para la cual se empleó la técnica de grupo focal, en 4 grupos de 10 integrantes cada uno. Se evidenció la presencia de elementos resilientes tales como: una actitud positiva y la percepción de crecimiento personal
\end{abstract}

Recibido: 2 de julio de 2020. Aprobado: 1 de junio de 2021

http://dx.doi.org/10.15359/rep.16-2.10

1 Este trabajo se realizó como una investigación con fines de titulación "Elementos resilientes utilizados por estudiantes de la Normal Rural Vasco de Quiroga de Tiripetío, Michoacán, México dentro de su movimiento.” de la Maestría en Psicología Clínica de la Universidad De Morelia, Se realizó entre los meses de agosto 2018 a noviembre del de 2019. No contó con ningún tipo de financiamiento.

2 Licenciada en psicología, maestrante en psicología clínica de la Universidad de Morelia. https://orcid.org/0000-0002-7388-2994

3 Doctora en psicología, profesora investigadora de la Universidad Michoacana de San Nicolás de Hidalgo, Michoacán, México. https://orcid.org/0000-0003-4488-586X 
y de logro de aprendizajes a partir del movimiento estudiantil. Esto permite concluir que este tipo de movimientos generan aspectos positivos en los estudiantes, por lo que es importante realizar investigaciones poniendo énfasis en el conocimiento de estos fenómenos no solo como movimientos sociales o políticos sino como promotores de recursos psicológicos y del potencial humano.

Palabras clave: Formación docente, movimiento estudiantil, resiliencia

\begin{abstract}
The aim of this paper is to describe the resilient resources that the students of Escuela Normal Rural Vasco de Quiroga, Michoacán, México use in their student/social movement and in their professional training. This research was carried out through a qualitative methodology, of the phenomenological kind, with the participation of 40 male students, using the focus group technique in 4 groups of 10 members each. The findings show the presence of such resilient elements as a positive attitude and the perception of personal growth and learning achievement from the student movement. This allows us to conclude that this type of movement generates positive aspects in students, which points to the importance of carrying out research emphasizing the knowledge of these phenomena not only as social or political movements but as promoters of psychological resources and human potential.
\end{abstract}

Keywords: resilience, student movement, teacher training

\title{
Introducción
}

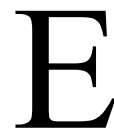

ste artículo aborda el tema de la resiliencia que desarrollan los estudiantes de la Escuela Normal Rural Vasco de Quiroga en Tiripetío, Michoacán, México, cuya permanencia se ha visto cuestionada en los últimos años por el Gobierno, lo cual ha dado lugar a una serie de protestas por parte de los estudiantes de la misma. En diversas ocasiones, estas manifestaciones han terminado en enfrentamientos con la autoridad municipal, estatal y federal. 
La presente investigación no trata de enfocarse en la emisión de un diagnóstico, o en la búsqueda de patologías que etiqueten y estigmaticen a este grupo. Por el contrario, intenta enfatizar la exploración de un fenómeno social desde la psicología humanista, centrándose en el análisis de los elementos resilientes que desarrollan los estudiantes de la Normal Rural para continuar con sus estudios a pesar de la incertidumbre y el ambiente hostil que muchas veces enfrentan.

Por este motivo, se planteó desde una perspectiva cualitativa, a través de la realización de una serie de grupos focales con los estudiantes, como un estudio de caso, entendiendo por este a toda la institución educativa, tal como lo plantean Rodríguez, Gil y García (1999).

Schmelkes (2003) plantea que, en México, la educación superior es vista como un privilegio que solo pocos pueden obtener; únicamente tres de cada diez jóvenes entre 18 y 24 años de edad tienen espacio en el nivel de la Educación Superior. Sin embargo, estas cifras son "engañosas", pues existe una diferencia en cuanto a las oportunidades educativas en el país. De acuerdo con esta autora, no son las mismas oportunidades en la Ciudad de México que en Chiapas, un estado con mayor pobreza. Se puede decir entonces, que las oportunidades de ingresar a este nivel educativo, por parte de estudiantes provenientes de estados vulnerables, se presenta como nula. Sin embargo, dentro de la historia de nuestro país encontramos una institución educativa, conocida como Escuela Normal Rural que fue creada con el fin de llevar educación a las comunidades marginadas, que tienen como base los ideales del progreso nacional mediante la educación pública (Arreola et al., 2012).

De la Corte (2000) postula que, para cada situación que acontece dentro de una población, es importante considerar el contexto sociocultural, económico, y psicológico de la región donde se sitúa, fechar estos fenómenos sociales y no pretender venderlos como universales, puesto que son locales y particulares, de ahí la pertinencia de hacer una investigación que se acerque a cada realidad que se esté tratando comprender.

En este orden de ideas, es necesario explicar que la Escuela Normal Rural en México fue creada en 1922, con la finalidad de llevar educación a las comunidades rurales marginadas. Tenía como base los ideales del progreso nacional mediante la educación pública, para dar paso a una educación libre del dominio del clero, una educación de México para los mexicanos. Se forman así, profesionistas con una visión 
de ayuda, no solo basada en "la enseñanza de conocimientos tradicionales a campesinos e indígenas, sino a la organización para su liberación" (Arreola et al., 2012). Es decir, que lleva 98 años formando profesores destinados a las comunidades rurales más pobres del país.

Sin embargo, en la actualidad solo se habla de estas normales para destacar actos reprobables para la sociedad como pintas, manifestaciones, retención y quema de vehículos, entre otros, conductas que, descontextualizadas, podrían hablar de patologías, al dejar de lado los motivos que han tenido los estudiantes de estas instituciones para optar por medios violentos de manifestación y lucha social.

De acuerdo con Poy (2016), la ofensiva en contra de las normales rurales se puede observar en el deterioro de sus instalaciones, los recortes financieros y las carencias en la plantilla académica, así como una disminución en el número de la inscripción. "En los pasados 30 años, las normales han perdido más de 200 mil estudiantes, es decir, $63.5 \%$ \%" (Poy, 2016, párr. 4). Este plan de "restructuración" tuvo el primer enfrentamiento violento el 19 de febrero del 2000, cuando la policía estatal realizó un operativo de desalojo en el centro de la ciudad de Pachuca y en la localidad de Mexe, Estado de Hidalgo, en donde estudiantes de la Escuela Normal Rural "Luis Villarreal" mantenían una huelga de hambre dentro de las instalaciones en demanda de más espacios para los alumnos de nuevo ingreso y aumento al presupuesto para el plantel. Se dio un enfrentamiento entre la policía y los alumnos, padres de familia y vecinos de la comunidad, para evitar el desalojo de la escuela (Camacho, 2015).

Desde entonces, el Gobierno mantuvo una estrategia de disminuir en el número de lugares ofertados para los aspirantes, hasta lograr la desaparición del plantel. Finalmente, en 2005, se entregaron las cartas de pasantes a los últimos licenciados egresados de la normal rural "Luis Villarreal" (Camacho, 2015). El cierre de esta escuela normal representa el ejemplo más claro de la estrategia del Gobierno, ya que la normal de Mexe es ahora un fragmento en la historia de las Escuelas Normales. Sin embargo, para los estudiantes normalistas es el recordatorio constante de que este mismo destino puede sufrir su institución educativa.

Ante estos eventos, se formó la Federación de Estudiantes Campesinos Socialistas de México, cuyo vocero Juan Daniel Torrez Vásquez afirma que, la supuesta modernización de los 17 planteles no es más que un pretexto para desaparecer las normales rurales (Mejía, 2016). 
De esta forma, se presenta una lucha por la defensa de las Escuelas Normales Rurales, llevada a cabo por los propios estudiantes normalistas. Un hecho que ha generado un gran impacto en nuestra sociedad, así como la indignación no solo del pueblo mexicano, sino también de los extranjeros, fue el evento ocurrido el 26 septiembre del 2014, con estudiantes de la Escuela Normal Rural "Raúl Isidro Burgos" ubicada en Ayotzinapa, Estado de Guerrero. Quienes se dirigían a su institución educativa, fueron detenidos por policías municipales, lo que terminó con la muerte de 3 estudiantes y la desaparición de 43 de ellos (Mosqueda et al., 2014).

En la opinión de Hernández (2012), la situación de las normales rurales en México es un reflejo de un conflicto de discursos educativos que va más allá de las escuelas y sus estudiantes: por un lado el discurso en favor de la modernización educativa y la calidad académica de acuerdo a parámetros internacionales y por otro, la tradición de la educación socialista en México, herencia de la Revolución Mexicana y de las ideas de la escuela rural que se intentaron implantar siguiendo el modelo cubano. En dónde la misión del egresado de esos planteles es, por un lado, conservar los conocimientos tradicionales de las comunidades campesinas e indígenas $\mathrm{y}$, por otro lado, ayudar a la organización política de estas. Como consecuencia de este desencuentro, se han generado una serie de sucesos violentos relacionados con las normales rurales.

El evento más reciente se presentó el 21 de junio del 2017, en la escuela Normal "Vasco de Quiroga" de Tiripetío, Estado de Michoacán. En el cual un estudiante fue herido de bala en un enfrentamiento con policías (Martínez, 2017).

En este sentido, la presente investigación pretende destacar los elementos resilientes con que cuentan los estudiantes de la Escuela Normal Rural "Vasco de Quiroga" y su potencial humano ante las situaciones de riesgo que viven por su enfrentamiento con las autoridades.

Por lo tanto, se plantea la siguiente pregunta de investigación: ¿Cuáles son los recursos resilientes que los estudiantes de la Escuela Normal Rural Vasco de Quiroga emplean dentro de su movimiento estudiantil, para seguir adelante con su formación profesional?

De tal forma, la presente investigación plantea como objetivo general, describir los recursos resilientes que emplean los estudiantes de 
la Escuela Normal Rural Vasco de Quiroga en su movimiento estudiantil y en su formación profesional.

De este, se desprenden los siguientes objetivos particulares:

- Identificar desde la psicología humanista los elementos resilientes de los estudiantes.

- Definir las estrategias que utilizan los estudiantes para su formación profesional.

- Explicar el papel de la resiliencia en el movimiento social de los estudiantes.

\section{Antecedentes}

La relación entre la resiliencia y la educación ha sido abordada por varios autores, entre ellos, Villalta y Saavedra (2010), quienes realizaron un estudio correlacional, para establecer en qué medida se asociaban estos logros educativos con la percepción de la cultura institucional, las prácticas de enseñanza en clase y la resiliencia tanto de alumnos como de profesores. Los autores concluyen que si bien existe una correlación alta entre las percepciones de cultura institucional y las prácticas de enseñanza; la correlación de estos dos factores con la resiliencia es moderada.

Otro estudio, realizado por Silas (2008), en 16 comunidades marginadas de México, pretendió explorar el significado que tiene perseverar en los estudios para los jóvenes de estas comunidades. El estudio se llevó a cabo con un enfoque cualitativo a través de entrevistas a 29 jóvenes, con la intención de conocer cuáles habían sido los actores y procesos, ya sean familiares, escolares o comunitarios, que influían o participaban en el éxito escolar, y la relación de estos con la teoría de la resiliencia. El autor concluye que los aspectos más significativos son, por un lado, el valor que la comunidad le da a la educación escolarizada $\mathrm{y}$, por otro, la presencia de personas significativas que han concluido sus estudios y sirven de ejemplo para el joven. Es decir, que se combinan dos aspectos, el impulso y el ejemplo.

Otra investigación se desarrolló en la comunidad maya en Yucatán, México, por Aguiar y Acle-Tomasini en el 2012, en donde se trabajó con un grupo de estudiantes mayas de secundaria que viven en condiciones de marginación social y pobreza, aunadas a problemas 
de violencia escolar, consumo de alcohol y embarazos no deseados, que, en ocasiones, conducen a la deserción escolar. Los autores concluyen que la promoción de las capacidades resilientes y los recursos personales durante los estudios de secundaria, pueden mejorar la adaptación al centro escolar, así como la situación personal y familiar de los adolescentes.

En estas investigaciones, se puede observar que, a pesar de lo sucedido, los estudiantes continúan asistiendo, formándose y culminando con sus estudios. Sin embargo, no se han encontrado estudios o investigaciones dirigidas a conocer los factores que los impulsan a seguir adelante, y los recursos que emplean para superar la adversidad.

\section{Marco teórico}

\section{Historia de las Escuelas Normales Rurales en México}

González (2017) realiza un análisis de la situación de las normales rurales, en el cual se puede ver como poco a poco se ha ido disminuyendo el presupuesto que reciben las escuelas de los gobiernos estatales y federales, en lo que el autor considera una estrategia gubernamental para la desaparición de las mismas, lo que se ha encontrado con la oposición de los propios estudiantes de las escuelas. Esta estrategia ha generado, a pesar de la oposición, una baja importante en las solicitudes de ingreso, que pasaron de 1350 en el 2012 a 390 en el 2016. Sin embargo, para entender la importancia de estas instituciones y su permanencia, es importante revisar su historia.

La Escuela Normal Rural Vasco de Quiroga se considera como la primera escuela de su tipo en México, esta formó parte de la primera universidad de América Latina, pues dependía, en sus inicios, de la Universidad Michoacana de San Nicolás de Hidalgo. Creada, en 1922 en el municipio de Tacámbaro Michoacán, con la finalidad de derrocar el dominio del clero, dando paso a lo que se denominó la escuela de México para los mexicanos, y el inicio del cambio, para llevar a las comunidades marginadas los beneficios de la educación fincada en el cumplimiento de la ley de la Constitución Política en México (Arreola et al., 2012).

De 1922 a 1925, tuvo como base el sistema de estudios Universitarios, pero carecía de la capacidad para crear el carácter de trabajo y de acción que debía poseer un legítimo maestro rural. En el periodo de 
1926 a 1930, la institución ya contaba con un plan de estudio propio que fue establecido por la Secretaría de Educación, en el cual las materias académicas que se impartían a los alumnos eran aquellas estrictamente necesarias para el desarrollo laboral de apoyo en las comunidades rurales. A partir de 1927, la escuela se organizó en la modalidad de internado exclusivo para varones, proporcionando hospedaje y alimentación a los estudiantes (Arreola et al., 2012).

En la actualidad, la escuela Normal se encuentra en la comunidad de Tiripetío Michoacán desde el año de 1959, en donde se da alojamiento a 560 estudiantes, provenientes no solo de Michoacán, sino de otros estados como Guerrero, Puebla, Veracruz, Guanajuato entre otros, bajo el mismo concepto (González, 2017).

\section{Resiliencia}

Resiliencia es un término retomado de la mecánica que indica la propiedad de la materia, que se opone a la rotura por el choque o percusión; Cyrulnik (2002) retoma este concepto dentro de la psicología para definir tanto la capacidad de resistencia al sufrimiento psicológico, como la posibilidad interior de la persona de recuperarse, es decir, la capacidad que posee el ser humano para soportar la adversidad, saliendo fortalecido y alcanzando un estado de equilibrio personal.

Otra definición la encontramos en Becoña (2006), el cual considera que la resiliencia es un rasgo psicológico, que es visto como un componente del self que capacita para el éxito en condiciones de adversidad, y que puede ser desgastado o, paradójicamente, reforzado por la adversidad. En este sentido el autor plantea que la resiliencia implica no solo un afrontamiento, sino también una transformación, un aprendizaje, y un crecimiento, que se mantienen aun después de superada la adversidad. Becoña (2006) explica que el proceso de resiliencia implica, ante todo, la exposición a una amenaza o evento adverso importante, por otra parte, que la persona lleva a cabo una adaptación positiva al impacto de ese proceso.

De acuerdo con Cyrulnik (2002), cuando se presenta una situación dolorosa, la naturaleza de este acontecimiento hiriente puede corresponder a todas las instancias de un aparato psíquico, ya sea este biológico, afectivo, o histórico. De tal forma que, a cada nivel, es posible que se desarrolle una resiliencia. La persona puede transformar el 
dolor del momento, para hacer de él un recuerdo glorioso o divertido, a través de un alejamiento emocional en donde entra en juego una serie de mecanismos de defensa capaces de disminuir el malestar provocado por la situación dolorosa.

Estos mecanismos son: la negación, el secreto, la angustia agresiva, la huida hacia adelante, la intelectualización, y la sublimación que permite, a través de la creatividad, transformar la situación dolorosa en arte, en humor, o bien, en altruismo y compromiso social (Cyrulnik y Anaut, 2016).

Otra forma resiliente de afrontar una situación es atribuir sentido al acontecimiento doloroso, lo cual permite modificar lo que se experimenta. Lukas (2003) refiere que:

No hay ninguna situación en la vida que carezca realmente de sentido, esto se deduce al hecho de que los lados aparentemente negativos de la existencia humana, especialmente la triada trágica formada por el sufrimiento, la culpa y la muerte también pueden adoptar la forma de algo positivo, de una exigencia si se tratan con la actitud y la postura correcta. (p. 23)

Por su parte, Frankl (1994), aunque no utiliza el término resiliencia, sí considera que el convencimiento de la persona de que su vida tiene sentido, en cualquier circunstancia, es clave para poder tener una visión positiva de la vida. Es a partir de esta resiliencia que el sujeto se logra sobreponer a las circunstancias y se erige como actor de su historia (Cyrulnik y Anaut, 2016).

Es decir, la resiliencia tiene que ver con aquello que permite orientar hacia la búsqueda de factores que ayudan a afrontar los desafíos de las crisis y a incrementar los recursos psíquicos y sociales para atravesar la adversidad con ingenio y creatividad (Del Pino et al., 2011); esta es una construcción propia y varía según los contextos y los momentos en que ocurre el evento a afrontar (Saavedra et al., 2012). De acuerdo con esto, Becoña (2006) establece una clasificación de "cuatro patrones para la resiliencia: el patrón disposicional, el patrón relacional, el patrón situacional y el patrón filosófico" (p. 129).

Según Becoña (2006), el patrón disposicional se define, en parte, por aspectos físicos o genéticos, como inteligencia, temperamento y 
salud, y en otra, a factores psicosociales como el autoconcepto o el sentido del self y la competencia personal o la autoeficacia.

El segundo, denominado relacional, analiza la influencia de los roles y las relaciones en la resiliencia, incluye aspectos intrínsecos de la relación, como la capacidad para identificar modelos positivos y establecer relaciones de confianza con ellos, darle algún sentido o significado a las experiencias y el aspecto extrínseco se refiere a la capacidad de confiar en el otro y mostrarse como alguien digno de confianza (Becoña, 2006).

El tercero, denominado situacional, describe la forma en que la persona se aproxima a las situaciones estresantes, e implica habilidades cognitivas como: la solución de problemas, la capacidad de acción, la evaluación realista de las consecuencias de las acciones y de la propia capacidad de respuesta, la habilidad para definir metas, el afrontamiento activo de los problemas y la posibilidad de reflexión. En este, se consideran también la creatividad, la curiosidad por explorar y buscar novedades (Becoña, 2006).

El último, se denomina patrón filosófico, y se relaciona con encontrar un sentido único y particular a la propia vida. También abarca las creencias personales, la capacidad de reflexión sobre uno mismo y sobre los eventos importantes. Así como la certeza de que la situación puede mejorar y la capacidad de encontrar un significado positivo a las experiencias desagradables (Becoña, 2006). Este concuerda con lo que Frankl (1991) denomina sentido de vida.

De acuerdo con Madariaga (2014), la resiliencia es un proceso que se construye en y desde la sociedad, aunque se manifieste en comportamientos individuales. En este sentido, la resiliencia no solo es la capacidad de soportar circunstancias adversas, sino también salir fortalecido de ellas y alcanzar un estado de equilibrio personal (Cyrulnik, 2002).

\section{Metodología}

Esta investigación se realizó a través de un enfoque cualitativo con un método fenomenológico, el cual no busca la representatividad o generalización de los resultados, sino la profundización en un fenómeno particular (Rodríguez et al., 1999). 


\section{Escenario}

La Escuela Normal se encuentra en la comunidad de Tiripetío Michoacán desde el año de 1959, en ella se da alojamiento y formación profesional para ejercer como profesor de primaria, a 560 estudiantes varones, provenientes no solo de Michoacán, sino de otros estados como Guerrero, Puebla, Veracruz, Guanajuato, entre otros, bajo el concepto de internado.

\section{Participantes}

Se llevó a cabo con la colaboración de 40 estudiantes de segundo, tercero y cuarto año de la Licenciatura en Educación Primaria, varones, las edades oscilan entre 19 a 24 años.

Con fines de identificación los discursos de los participantes, se codificaron de la siguiente forma: $\mathrm{P}$ (número consecutivo) Año escolar. Ejemplo: P1-4 (participante 1 de cuarto año).

\section{Técnica de recolección de información}

Se utilizaron 4 grupos focales, de 10 integrantes cada uno. El cual es descrito por Bonilla y García (2002) como una conversación entre un número reducido de personas sobre un tema en específico, que es conocido y trabajado en común y, de esta manera, conocer la percepción construida de manera colectiva. Se realizaron 5 preguntas con respecto a cómo los estudiantes han vivido su experiencia en la Escuela Normal. Dichas preguntas fueron:

- Dentro de los acontecimientos vividos en el ámbito educativo en el que te desenvuelves, ¿consideras que has podido sobrellevar los tiempos difíciles?, ¿por qué consideras esto?

- ¿Cuáles son los recursos que has empleado dentro de estos acontecimientos?

- ¿Cómo has empleado estos recursos?

- ¿Consideras que lo que ha ocurrido en el pasado te ha dejado algún aprendizaje?, ¿cuál sería ese aprendizaje?

- $\quad$ ¿Consideras que tu vida posee un significado y un propósito?, ¿cuál sería este? 


\section{Procedimiento}

1. Se planteó el proyecto de investigación, el cual fue aceptado por parte del Consejo estudiantil. Se garantizó la confidencialidad de datos personales de los participantes. Asimismo, se estableció que los resultados obtenidos de los grupos focales serían presentados en este artículo.

2. Desarrollo de los grupos focales. Se expone la primera pregunta y se entregan hojas blancas para desarrollar las respuestas, posteriormente se lleva a la socialización de cada una de las preguntas, esta misma acción se toma en cada grupo.

3. Análisis de las respuestas por categorías. La información brindada por los participantes, en los grupos focales, ha sido estudiada desde el análisis del discurso (AD). El AD se interesa, no tanto, por las características lingüísticas abstractas al uso de la lengua sino, ante todo, por la forma en la que el discurso presenta la realidad de los hablantes en función de los procesos de producción, distribución e interpretación del mismo (Karam, 2005). De esta forma, se intentó abordar aquello que las personas expresaron en el lenguaje que no está ceñido a las palabras ni al significado puntual de las mismas sino a la globalidad del discurso. En esta medida, Íñiguez (2003), indica que el AD trasciende el estudio de la forma lingüística, para ir más allá de la oración y de la frase.

\section{Consideraciones éticas}

Se realizó un oficio de solicitud a los directivos de la institución, asimismo se informó a los estudiantes que su participación sería voluntaria y que los datos personales no serían evidenciados en esta investigación.

\section{Resultados y discusión}

Si bien la investigación completa aborda el desarrollo de las cuatro categorías basadas en los patrones resilientes de Becoña (2006), descritos anteriormente, en este artículo solo se presenta el patrón situacional. Este se refiere a las habilidades cognitivas para enfrentar las situaciones que provocan estrés; es decir, a la capacidad para comprender la naturaleza, relaciones y cualidades de un fenómeno determinado y enfrentarlas de forma racional, a través de evaluar, de forma realista, 
tanto las propias capacidades como las consecuencias de las acciones (Sambrano, 2011). También, a la capacidad de definir metas específicas para afrontar, activamente, los problemas, y reflexionar sobre ellos (Becoña, 2006).

A continuación, se presentan transcripciones literales de fragmentos, de los discursos de los estudiantes durante el grupo focal, en los cuales se evidencian las habilidades cognitivas:

\section{Habilidades para solucionar los problemas}

P2-4a: Las herramientas fundamentales o eso considero yo son la serenidad, asi como buscar la mejor solución a los conflictos que se presenten, es decir, pensar con la cabeza fría y tratar de no dejarse llevar por los impulsos, teniendo como base un buen plan a seguir contemplando todos los lados de la operación, sin dejar de lado el constante diálogo con las autoridades competentes.

P8-4 : El ser inteligente, es decir, el saber actuar ante las situaciones de peligro. Observar tu entorno te permite hacer un plan de acción, logrando de esta manera la mejor actuación ante estas situaciones, es decir ante cada uno de los diversos movimientos a los que nos enfrentamos.

P6-4 ${ }^{a}$ : Cada movimiento tiene su propia firma es decir cada movimiento requiere de su propia planeación, de su propia estrategia para buscar la mejor solución, por lo que se requiere de un plan de acción diferente a cada movimiento.

Estas acciones corresponden al concepto que Cyrulnik (2002) denomina temperamento personal, es decir, la capacidad adaptativa que se produce debido a la adquisición de recursos internos impresos en el temperamento, desde las primeras interacciones a temprana edad. Estos explican la forma de reaccionar frente a las agresiones de la vida, las cuales crean guías de desarrollo que posibilitan la resiliencia. Por su parte, Sambrano (2011), refiere esta capacidad dentro de lo que ella denomina creatividad, la cual la define como un estado de consciencia que permite generar una red de relaciones para identificar, plantear, producir y resolver problemas de manera relevante y original. Asimismo, describe que las personas creativas son inteligentes, e implica tener una autoestima alta y una profunda motivación interna para cambiar las cosas, al respecto encontramos que los estudiantes, en específico, aportan. 
P5-3 ${ }^{\mathrm{a}}$ : La búsqueda de estrategias didácticas para fortalecer el conocimiento de los niños dentro de una escuela primaria, para que de esta manera, se pueda mejorar la educación y el desarrollo de los alumnos y de la comunidad en sí misma. Por medio de la preparación continua, poniendo en práctica los valores, es aquí donde encontramos una motivación encaminada a la búsqueda del cambio.

\section{Capacidad para la acción frente a una situación}

A esta planeación, se entrelaza la siguiente habilidad: acción frente a la situación. Pues como se puede observar, una cosa es planear y la otra es actuar. En este sentido, encontramos varios ejemplos que nos dan cuenta de la presencia de esta habilidad en los estudiantes P5-3 ${ }^{a}$ : Realizar actividades deportivas o alguna otra actividad de una manera responsable.

P8-3 ${ }^{a}$ : Algo que siempre funciona para mi es tratar de distraerme al principio, dejar todo en calma para después buscar una posible solución, es decir, si tratas de solo enfocarte en el problema, solo se estará más estancado. Para distraerme, por lo general, trato de viajar, visitar mi familia y desconectarme de esas situaciones por un tiempo.

P4-4 : El valor para enfrentar los diversos movimientos y de esta manera me he dado cuenta que los conocimientos no solo se adquieren en un aula de clases, y de esta manera poder trasmitir estos conocimientos a los niños.

Desde el punto de vista de Rousseau (2012), refiere esta habilidad como estrategias de ajustes activos. Es decir, actuar o pasar a la acción para mejorar la situación. A esto, Rousseau (2012) agrega un término sin el cual no se presentaría la habilidad de planeación, ni mucho menos la acción. Este es, pues, el término espíritu combativo que se refiere a mantenerse firme y luchar incansablemente para conseguir los objetivos.

\section{Habilidad de hacer una evaluación realista de la propia capacidad para actuar}

Se refiere a la necesaria introspección para que la persona sea capaz de observarse honestamente y reconocer sus habilidades $y$ limitaciones. Para Rousseau (2012), una parte fundamental en el sentimiento de eficacia personal se sitúa, de forma ligera, por encima de 
las capacidades reales, lo cual fomenta que la persona afronte tareas difíciles y acumule experiencias nuevas.

En esta, encontramos las palabras de los estudiantes que refieren:

P7-4 ${ }^{a}$ : Conocer de qué estás hecho, posibilita la oportunidad de buscar la mejor solución a las confrontaciones con el gobierno.

P10-3a: Cuando uno se llega a conocer bien y sabe que puede hacer y que no puede hacer le permite buscar ayuda en aquellas áreas que no es tan bueno y de esta manera puede con ayuda de los demás formar un gran plan de acción a cada una de estas represiones. Por ejemplo, si no es más bueno para comunicarse verbalmente y otro para la escritura, cada uno aporta algo y de ahi surge un plan bueno.

P3-2a: Yo considero que la mayor estrategia proviene de cuando tú te conoces y sabes cuáles son tus defectos, y de esta manera, sabes con que sí cuentas y con que no, pero no es que no cuentes sino más bien, es como en qué puedes crecer más.

\section{Evaluación realista de las expectativas y consecuencia de esa acción}

En esta evaluación, tenemos varios ejemplos.

P7-2 ${ }^{a}$ : No dejarnos llevar por cualquier cosa, saber aprovechar muy bien las oportunidades y asi que todo salga bien.

P10-3a': Observar las ventajas que tiene el diálogo para prevenir todos estos acontecimientos.

Para Rousseau (2012), los factores de protección están relacionados entre sí e influyen los unos en los otros. Al tener esto en cuenta, podemos observar que desde la planeación hasta la ejecución se presentan eslabones que los unen a un mismo proceso.

\section{Búsqueda de novedad, curiosidad, naturaleza exploratoria y creatividad}

P5-3 $3^{a}$. Considero que he podido sobrellevar los tiempos dificiles, respecto a realizar actividades deportivas o alguna otra actividad, de una manera responsable.

P2-4: Los recursos con los que yo cuento son habilidades en el área deportiva, así como destacar en el ámbito académico, sin embargo, las herramientas fundamentales o eso considero yo son la serenidad, así como buscar la mejor solución a los conflictos que se 
presenten, es decir pensar con la cabeza fría y tratar de no dejarse llevar por los impulsos

P1-2 ${ }^{a}$ : De igual manera la práctica de un deporte o actividades productivas de la vida diaria ayudan a seguir adelante.

P10-3․ Otra sería por medio de pintar murales, asi como informar a la gente de lo que sucede, realmente en la institución.

P9-4a: Suelo pintar la historia de mi Normal, es decir dibujo, escribo pero sobretodo trato de moldearme a las circunstancias que enfrentamos.

P9-2 ${ }^{a}$ : Dentro de mis recursos considero que la espontaneidad, la creatividad me han ayudado a ser flexible e ir planeado, observado el ambiente en el que me desenvuelvo, así como los círculos de estudio nos permiten tener una mirada más detallada de la situación que estamos enfrentado, pues con los conocimientos, habilidades podemos tener un mejor plan de acción.

Para Sambrano (2011), la creatividad da poder, esto es porque la imaginación, la intuición y los deseos de cambiar actúan como motores, en los momentos que las transformaciones (personales o sociales) son necesarias. Estas actividades, Rousseau (2012) las define como un mecanismo de defensa en el cual se orienta la pulsión inaceptable hacia una actividad intelectual, espiritual, profesional, artística, deportiva o socialmente valoradas, mientras que Cyrulnik (2002) plantea la importancia que juega la creatividad, a través de la cual se puede liquidar nuestro sufrimiento, consciente o inconscientemente y se puede expresar lo que de otro modo no se podría.

\section{Perseverancia}

P10-2: Las fuerzas, las ganas de ser una mejor persona cada día, centrarme en mis estudios para ser un buen maestro.

P10-3a' Cada día cuando me levanto pongo todo mi empeño para aprender, así como dedico horas extras a mis clases para estudiar para aprender nuevas cosas.

Así como locus de control interno.

P6-3 ${ }^{a}$ : Sí, porque mis sanos estudios me llevan a sobre salir durante los momentos difíciles al igual que mis ganas de que los niños tengan la oportunidad de estudiar y que esta sea de calidad. 
P3-4a: En cuanto a la formación que obtuve dentro de la normal rural presencie diferentes acontecimientos en la vida diaria dentro de la institución pues durante 4 años pase más tiempo en la institución que en mi casa, donde cada ciclo escolar se presentado un nuevo reto dentro y fuera de la institución y así reconociendo como afrontar cada uno de esto para de esta manera continuar con mi camino para llegar a mi objetivo, pues como se ha observado altos funcionarios intentan truncar la formación.

Para Rousseau (2012), esta habilidad está acompañada de la habilidad de tomar iniciativa; Erikson (1982) define que esta habilidad se adquiere entre los 3 y 6 años de edad. En este periodo, el niño intenta tener nuevas experiencias y desarrollar otras habilidades, e intenta tener un equilibrio entre su deseo de hacer cosas y la culpa de haber ido, a menudo, demasiado lejos, así mismo está unida a la confianza en uno mismo.

\section{Humor}

Al igual que la creatividad y la fantasía, les permite construir un mundo alterno al que están viviendo, esto no quiere decir que escapen de su realidad, solo se adaptan y la convierten, para hacerla menos dolorosa, como lo podemos observar en los siguientes comentarios.

P4-4 ${ }^{a}$ : A veces reir de la situación ayuda a que el tiempo pase y que tú espera, o la incertidumbre de no saber lo que te espera ayuda a sacar un poco tu miedo.

P2-3': Uno puede ver la vida como un chiste es decir hay chistes buenos, pero también hay chistes malos, por eso trato de que cada cosa en vida pueda tener algo cómico que me haga más llevadera mi estancia en la Normal, es decir si veo las cosas siempre como fatalidades, desgracias serán eso para mí y para mis compañeros, siempre trato de que estos rían.

P7-2: Una vez que la situación pasa, y te das cuenta de lo que paso, de a cara de susto del otro, de las caídas, corretizas, uno se muere de la risa, es como una forma de expresar el miedo, el dolor, el sufrimiento que muchas veces pasamos en los movimientos.

Como se puede observar a partir de estos discursos, los estudiantes consideran que poco a poco han logrado salir adelante, con base en distintas estrategias, actividades y esfuerzo, etc. Sin embargo, también mencionan que la escuela les ha brindado herramientas para enfrentar 
los problemas pues se afirma que siempre hay una solución a cada problema.

Se habla de un análisis tomando en cuenta la realidad no solo de la institución sino del país en general, de esta manera lo que los estudiantes refieren es tener un plan de acción, así como un plan de emergencia a cada situación que se esté enfrentando, sin dejar de lado el establecimiento del diálogo con las autoridades competentes, en este también se incluye las habilidades de expresión de los estudiantes.

Por otra parte, la responsabilidad, creatividad, capacidad de escucha y la búsqueda de estrategias para resolver problemas, no solo les ayudan en el desarrollo de su movimiento social, sino que también favorecen su formación como profesores de primaria. Lo que se busca con esto, desde el punto de vista de los estudiantes, es crear en sus alumnos una mirada realista de la sociedad en la que vivimos, así como que estos pequeños sean críticos, analíticos.

A partir de las respuestas de los alumnos de $2 .^{\circ}, 3 .^{\circ}$ y $4 .^{\circ}$ grado de la escuela Normal Rural Vasco de Quiroga, se confirmó la presencia de elementos resilientes en esta comunidad educativa. Se observó, además, que los sentimientos a los que se enfrentan día con día se encuentran inmersos de un sentido. Tal como lo propone Frankl (1994), al explicar que, para que la persona pueda adoptar una visión positiva de su vida, tiene que estar convencida de que, independientemente de las circunstancias a las que se enfrente, su vida tiene un sentido. Así los estudiantes de la normal, viven inmersos en una causa más importante que ellos mismos: mantener abiertas y funcionando las normales rurales, en concordancia con los principios para los que fueron creadas. Y esto les lleva a afrontar y superar las dificultades y presiones de su entorno. 


\section{Conclusiones}

Al hablar de la normal de Tiripetío, nos encontramos con comentarios enfocados a identificar el lado patológico de la población estudiantil, es decir, se descontextualiza y se juzga a aquellos fenómenos sociales que son locales y particulares, como universales (De la Corte, 2000); sin embargo, Carvajal (2018) habla de una psicología positiva, la cual tiene por objetivo el descubrimiento y la promoción de los factores que permiten al individuo y a las comunidades prosperar.

Como se puede observar, los eventos a los que los estudiantes se han enfrentado, modificaron sus vida, y les permitieron, tal como explica Carvajal (2018), entender el mundo de una forma diferente y construir un sistema de valores acorde a la realidad en que viven. De tal forma que, ellos no pierden la fortaleza para desarrollar al máximo sus potencialidades.

A partir de los resultados, se concluye que existen elementos resilientes en los estudiantes de la Escuela Normal Rural Vasco de Quiroga. Entre ellos, se destaca el patrón situacional, en los alumnos, pues como menciona Frankl (1991), si no está en tus manos cambiar una situación que te produce dolor, siempre se podrá escoger la actitud con la que se afronta. Precisamente, este patrón explica la manera en la que los alumnos le hacen frente. Aunque ellos reconocen que no hay una solución fácil a la realidad, se identificó una actitud positiva con experiencias satisfactorias de crecimiento, por medio de la presencia de elementos tales como, las habilidades de solución de problemas, la capacidad para la acción frente a una situación, la competencia de hacer una evaluación realista de la propia aptitud para actuar(autoconocimiento) y de las expectativas o consecuencias de esa acción.

Estas habilidades juegan un papel determinante en el afrontamiento de circunstancias, ya que el confiar en sus propias capacidades les ha abierto un sinfín de posibilidades de solución.

Esta investigación muestra que la resiliencia se puede desarrollar y trasmitir. Sin embargo, aún existen vacíos en la forma de medirla o evaluarla, que requieren mayor investigación. De ahí la importancia de seguir haciendo investigación que permita un mayor conocimiento sobre estos fenómenos, que más allá de ser movimientos sociales o políticos implican hablar de recursos psicológicos y del potencial humano. 


\section{Referencias}

Aguiar, E. y Acle-Tomasini, G. (2012). Resiliencia, factores de riesgo y protección en adolescentes mayas de Yucatán: elementos para favorecer la adaptación escolar. Acta Colombiana de Psicología, 15(2), 53-64.

Arreola, M., Canchola, M., Ávila, G., Árciga, M., Flores, E., Negrete, M. y Guerrero A. (2012). Escuela Normal Rural "Vasco de Quiroga". 90 años de historia. Morevallado.

Becoña, E. (2006). Resiliencia: definición, características y utilidad del concepto. En Revista de Psicopatología y Psicología Clínica, 11(3), 125-146. https://doi.org/10.5944/rppc.vol.11. num.3.2006.4024

Bonilla, P. y García, C.(2002). Las técnicas de investigación cualitativa. Universidad Intercontinental.

Camacho, C. (8 de febrero de 2015). El Mexe hace 15 años. La Crónica de hoy en Hidalgo. http://www.cronicahidalgo.com/2015/02/ el-mexe-hace-15-anos/

Carvajal, M. (2018). Psicología positiva: camino para la prevención y la adversidad. Trabajo presentado en el XXX Aniversario de la Unidad Académica de Psicología de la Universidad Autónoma de Zacatecas, Zacatecas México.

Cyrulnik, B. (2002). Los patitos feos. La resiliencia: una infancia infeliz no determina la vida. Gedisa.

Cyrulnik, B y Anaut, M. (2016). ¿Por qué la resiliencia? O que nos permite reanudar la vida. Gedisa.

De La Corte, L. (2000). La psicología de Ignacio Martín-Baró como una psicología social crítica. Una presentación de su obra. Revista Psicología General y Aplicada, 53(3), 437-450. https://dialnet. unirioja.es/servlet/articulo?codigo $=2357055$

Del Pino, M. A., Bustamante, H. A., Ojeda, S. H., Fernandez, D. A., Romano, C. C., \& Romano, C. S. (2014). Vulnerabilidad adolescente: factores que favorecen la resiliencia en los jóvenes de la localidad. Informes Cientificos Técnicos - UNPA, 3(3), 62-80. https://doi.org/10.22305/ict-unpa.v3i3.38

Erickson, E. (1982). El ciclo vital completado. Paidós.

Frankl, V. (1991). El hombre en busca de sentido. Herder.

Frankl, V. (1994). La voluntad de sentido. Herder. 
González Báez, M. (2017). La reforma educativa en la formación pedagógica del normalista rural [Tesis de maestría no publicada]. Instituto Michoacano de Ciencias de la Educación, Morelia.

Hernández, L. (2012). El asesinato de Minerva: la batalla por el normalismo rural. El cotidiano, 176, 19-33. https://www.redalyc.org/ pdf $/ 325 / 32525230003$.pdf

Íñiguez, L. (2003). Análisis del discurso. Manual para las ciencias sociales. UOC.

Karam, T. (2005). Una introducción al estudio del discurso y al análisis del discurso. Globlal Media Journal, 2(3), 34-50. https://www. redalyc.org/pdf/687/68720305.pdf

Lukas, E. (2003). Logoterapia: la búsqueda de sentido. Paidós.

Madariaga, J. (2014). Nuevas miradas sobre la resiliencia. Barcelona: Gedisa.

Martínez, E. (22 de junio de 2017). Reacciona normalista de Tiripetío herido de bala. La Jornada, 26. https://www.jornada.com. $\mathrm{mx} / 2017 / 06 / 30 /$ estados/026n3est?partner=rss

Mejía, F. (2 de julio de 2016). SEP busca desaparecer 17 escuelas normales rurales, acusan dirigentes de la Federación de Estudiantes Campesinos Socialistas de México. Milenio. http://www.milenio. com/politica/SEP-desaparecer-normles-rurales-acusan-escuelas-cierre-estudiantes_0_679132114.html

Mosqueda, L., Ceceña, A. y Durán, J. (2014). ¿Es crimen de Estado? Indignación internacional. La responsabilidad en la violación de los derechos humanos en Ayotzinapa, Iguala, Guerrero, México. Internaciones, 2(6), 113-128. https://doi.org/10.32870/ in.v2i6.6859

Poy, L. (14 de marzo de 2016). Escuelas Normales en el abandono oficial. La jornada. http://www.jornada.unam.mx/2016/03/14/ politica/002n1pol

Rodríguez, G., Gil, J. y García, E. (1999). Metodología de la investigación cualitativa. Aljibe.

Rousseau, S. (2012). La resiliencia: vivir feliz a pesar de. Obelisco.

Saavedra, E., Castro, A. y Saavedra, P. (2012). Autopercepción de los jóvenes universitarios y resiliencia: construcción de sus particularidades. Katharsis: Revista de Ciencias Sociales, 14, 89-105. https://dialnet.unirioja.es/servlet/articulo? codigo $=5527374$ 
Sambrano, J. (2011). Resiliencia: transformación positiva de la adversidad. Caracas: Alfa.

Schmelkes, S. (2003). Educación superior intercultural: el caso de México. Encuentro Internacional de Intercambio de Experiencias Educativas. Guadalajara, México.

Silas, J. (2008). ¿Por qué Miriam sí va a la escuela? Resiliencia en la educación básica mexicana. Revista Mexicana De Investigación Educativa, 13(39), 1255-1279. http://www.scielo.org.mx/scielo. php?script=sci_arttext\&pid=S1405-66662008000400010\&ln$\mathrm{g}=\mathrm{es} \&$ tlng=es

Villalta, M. y Saavedra, E. (2010). Cultura escolar, prácticas de enseñanza y resiliencia en alumnos y profesores de contextos sociales vulnerables. Universitas Psychologica 11(1), 67-78. https://doi. org/10.11144/Javeriana.upsy11-1.cepe 